\title{
Caffeine Increases Vertical Jumping Height in Young Trained Males Before But Not After a Maximal Effort Strength Training Session
}

\author{
Daniel S. Tangen ${ }^{1} \cdot$ Stian R. Nielsen ${ }^{1} \cdot$ Kristoffer J. Kolnes ${ }^{1,2} \cdot$ Jørgen Jensen ${ }^{1}$
}

Received: 23 September 2019 / Accepted: 4 March 2020 / Published online: 9 April 2020

(c) The Author(s) 2020

\begin{abstract}
The aim of the present study was to investigate the effect of caffeine on vertical jumping height in rested condition and after a heavy strength training session. Six well-trained young males with experience in jump and strength training were included in this double-blinded, randomised study with cross-over design. Caffeine ( $3 \mathrm{mg} / \mathrm{kg}$ body weight) or placebo were ingested 45 min prior to the jump tests. Jumping was performed on a force platform and vertical jumping height was calculated. After a standardized warm up, participants performed jumping series consisting of three maximal jumps with $30 \mathrm{~s}$ rest between jumps followed by five maximal jumps with $7 \mathrm{~s}$ rest between jumps. The participants performed a heavy strength training of the leg muscles (leg press: $3 \times 15$ reps) and the jumping series was repeated immediately after $(30 \mathrm{~s})$, and after 5 min and 15 min recovery. Caffeine increased the maximal vertical counter movement jump height $(P \leq 0.05)$ and mean value of the 5-jump sequence prior to the strength training. Caffeine increased jump height by $2.2 \mathrm{~cm} \pm 0.5 \mathrm{~cm}$ at the first jump. Blood lactate after the strength training increased to $6.97 \pm 1.20$ and $7.77 \pm 0.54 \mathrm{mmol} / \mathrm{L}$ in PLA and CAF, respectively $(P=0.19)$. The jump height was reduced by $8 \mathrm{~cm}$ after the strength training. There were no differences in jump height after ingestion of caffeine or placebo immediately after the strength training session or in the recovery period, but blood lactate in the recovery period was higher in CAF compared to PLA (ANOVA; $P<0.05$ ). Conclusion: Caffeine increased the vertical jump height in the resting state. However, after a maximal effort strength training session the positive effect of caffeine was no longer significant.
\end{abstract}

Keywords Lactate $\cdot$ Fatigue $\cdot$ Recovery $\cdot$ Force-velocity $\cdot$ Athletes

\section{Introduction}

Caffeine has ergogenic effect on many types of exercises, where physiological demands are quite different. The ergogenic effect of caffeine is best documented in aerobic exercise and many studies have reported improved performance after intake of caffeine [16, 30-32]. Benefits of caffeine has also been documented in exercise, where anaerobic energy contribution is high such as Wingate test $[6,13]$ and caffeine intake increases maximal voluntary strength [34]. A

Jørgen Jensen

jorgen.jensen@nih.no

1 Department of Physical Performance, Norwegian School of Sport Sciences, Sognsveien 220, Ullevål Stadion, PO Box 4014, 0806 Oslo, Norway

2 Present Address: Steno Diabetes Center Odense, Odense University Hospital, 5000 Odense C, Denmark meta-analysis concludes that caffeine increases MVC and muscular endurance [39].

Caffeine is a small molecule (1,3,7-trimethylxanthine) which binds and regulates several proteins [9]. Caffeine binds with high affinity to adenosine receptors and inhibits adenosine signalling $[9,40]$. However, caffeine has many actions and inhibits PI 3-kinase [8, 19], phosphodiesterase [9], glycogen phosphorylase a [26] and stimulates $\mathrm{Ca}^{2+}$ release [33]. Tarnopolsky and Cupido showed that caffeine increased force production during nerve stimulation at physiological concentration in humans, indicating that caffeine has a direct effect on skeletal in humans [35].

Adenosine receptors are widely distributed and expressed in most tissues. Adenosine receptors are highly expressed in the brain and there are strong evidences for that caffeine improves performance via effect on the nervous system [5, 9]. Caffeine influences many cell type/areas in the brain that could potentially contribute to improve performance [23]. In sport where large power production is crucial for 
performance, activation and motor neuron recruitment need to be high. It has been shown that caffeine ingestion increases spinal excitability [38], and voluntary activation and strength [2]. Furthermore, it has been shown that caffeine improves the force-velocity relationship and muscle fibre conduction velocity [1], which will be beneficial for power production and jumping heights.

Recently, some studies have also shown that caffeine improves jumping capacity $[3,24,28]$. Perez-Lopez et al. found that caffeine increased performance at a number of tests evaluating explosive strength including jump height [24]. It has also been shown that caffeine increases jumping height in volleyball players [41] and basketball players [25].

A key question is whether caffeine improves performance after fatiguing exercise. Caffeine intake improves performance and blood lactate is normally higher after exhaustive exercise after intake of caffeine compared to placebo [30-32]. Furthermore, the higher effort after intake of caffeine, compared to placebo, resulted in higher plasma creatine kinase (CK) the day after caffeine intake prior to the performance test [32]. It has also been reported that caffeine improves power during the first three sprints, whereas no effect was observed during the following seven sprints [12]. Therefore, caffeine intake may improve performance resulting in more extensive muscular stress, which will hamper performance later on. Therefore, it will be important to know whether caffeine can improve jumping performance in the early recovery after maximal effort strength training.

The aim of the present study was to investigate the effect of caffeine on maximal vertical jumping height and performance in a 5-jump sequence in young strength-trained males. Furthermore, we aimed for the first time to investigate the effect of caffeine on jumping performance in the early phase of recovery after a short maximal effort strength training series of the leg muscles.

\section{Materials and Methods}

Six healthy males with experience in strength and jump training completed the study, which was randomised, double-blinded with cross-over and balanced design. Participants were informed about the study and completed a health questionnaire. Exclusion criteria were known disease in heart or inability to perform heavy strength training or repeated maximal effort jumping. The study was performed as a student project without approval from Ethic committee. The study was conducted according The Declaration of Helsinki, and participants were informed about the project before they gave their informed written consent to participate and could withdraw at any time. Physical characteristics of the participants are shown in Table 1.
Table 1 Anthropometric characteristic and leg strength of participants

\begin{tabular}{lllll}
\hline Weight $(\mathrm{kg})$ & Height $(\mathrm{cm})$ & Age (years) & 1RM $(\mathrm{kg})$ & 15RM $(\mathrm{kg})$ \\
\hline $89.9 \pm 3.4$ & $183.5 \pm 2.6$ & $24.3 \pm 0.4$ & $294.2 \pm 21.4$ & $219.2 \pm 12.8$ \\
\hline
\end{tabular}

Leg strength is described as the load (kg) for 1RM and 15RM in back laying leg press

Data are mean \pm SEM. $N=6$

\section{Study Design}

The goal of the study was to investigate the effect of caffeine on jumping performance in the resting state and during the recovery period after a heavy strength training session of the leg muscles (leg press). The study included four test days within 2 weeks. On the first test day, anthropometrics data were collected, before one-repetition maximum (1RM) in leg press was tested and the jump series were practised. On the second test day, participants conducted familiarization training to find the correct weight that was going to be used during the strength training (15RM) and practised the jump series again.

The two main test days were separated by 7 days, and participants were not allowed caffeine containing drinks for the last $48 \mathrm{~h}$ before the tests and no exercise was allowed the last $24 \mathrm{~h}$ prior to the tests. On the first day, half of the group received caffeine and the other half placebo. On the second test day the groups were reversed. The study was double-blinded. Caffeine ( $3 \mathrm{mg} / \mathrm{kg}$ bodyweight) was mixed with FunLight raspberry (Stabburet 711-1411 Kolbotn) and placebo was FunLight raspberry without caffeine as in our previous studies [30]. The two drinks were given $15 \mathrm{~min}$ after arrival and $45 \mathrm{~min}$ before the start of the testing.

\section{Jumping Series}

Counter movement jump (CMJ) performed on a force platform and jumping height was calculated. The test subjects were instructed to keep their hands on their hip during the jumping phase. The subjects chose the optimal angle in the knee during the counter-movement jump. The counter-movement jump sequence included three single maximal jumps with $30 \mathrm{~s}$ rest between jumps, followed by a sequence of five jumps separated by $7 \mathrm{~s}$ rest (Fig. 1b).

\section{Pre-testing}

On the first pre-test day, the subjects tested their $1 \mathrm{RM}$ in back laying leg press. The 1RM testing were performed with a knee angle of $75^{\circ}$ measured with a goniometer. To control the movement angle in the leg press, a stopper was 
$\mathbf{a}$

Protocol

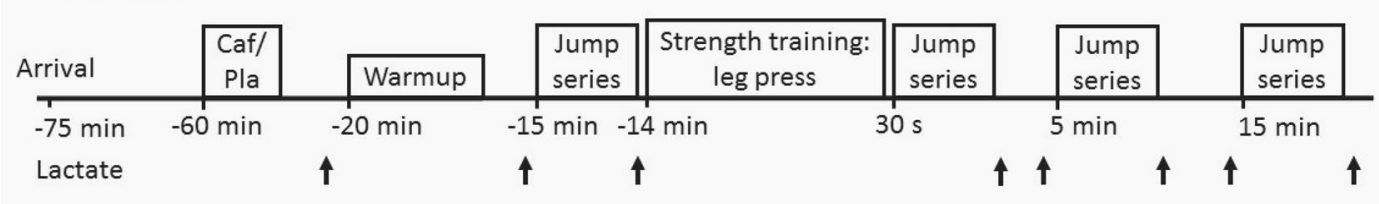

b

Jump series

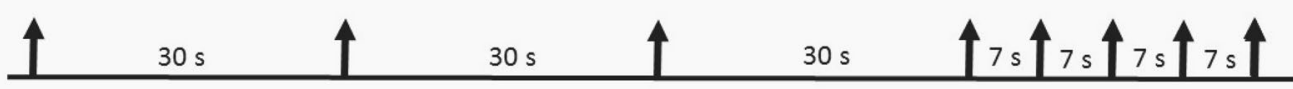

Fig. 1 Schematic overview of the design of the study and the jumping series. a Overview over the protocol on the test days. b Overview over the jumping series completed before and three times after the strength training session

adjusted individually to ensure a knee angle of $75^{\circ}$ in the leg press. Before testing 1RM, the subjects did 5 min of general warm up on a stationary bike and four sets of leg press with increasing weights (10, 6, 4 and 2 repetitions). To define $1 \mathrm{RM}$ the subjects performed single repetitions with increasing weight until they were unable to lift the weight. The $1 \mathrm{RM}$ was determined within four repetitions. After determination of $1 \mathrm{RM}, 15 \mathrm{RM}$ was estimated as $70 \%$ of $1 \mathrm{RM}$. The participants completed 15RM with the calculated load, and the weight was adjusted to correspond to 15RM based on rate of perceived exertion, according to Borg's CR10 scale for strength [4].

On the second day of pre-test, the subjects practised the test protocol used to study the effect of caffeine on jumping height. Participants performed the complete test procedure as used at the main test, but without caffeine and capillary blood samples.

\section{Main-Test}

The subjects arrived the test facilities 1 hour before the test started. After 15 min of rest, caffeine or placebo was given. The participants rested another 30 min before warmup started. Lactate was measured in capillary blood before warmup (descried below). The warmup consisted of 4 min easy jogging and 3-4 "sprints" with increasing speed ( 40 m). Approximately 1 min after the warmup, a new lactate measurement was performed. The first jump series was performed 45 min after intake of caffeine or placebo. After the first jump sequence, capillary lactate was measured.

Prior to the strength training session, participants performed specific warm up in leg press consisting of ten repetition at $40 \%$ of $1 \mathrm{RM}$ and eight repetitions at $60 \% 1 \mathrm{RM}$.
After the warmup, subjects performed three sets of dynamic leg press at 15RM. Each new set started every $3 \mathrm{~min}$. Immediately after the last repetition of the leg press, the subject walked over to the force plate and started the first jump sequence exactly $30 \mathrm{~s}$ after finishing the leg press. After the first single jump and after the jump sequence, lactate was measured. Lactate was also measured before and after jump series $5 \mathrm{~min}$ and $15 \mathrm{~min}$ after the strength training.

\section{Force Platform}

Counter-movement jumps were performed on a force platform (AMTI LG6-4-1 Amplifier AMTI SGA6-3 AMTI, 176 Waltham Street, Watertown, MA 02472, USA). The signals were filtered through a $1050 \mathrm{~Hz}$ low pass filter and transferred to a computer and jumping height was calculated [37]. Jumping height was calculated from the impulse during take-off phase.

\section{Blood Lactate}

Lactate was measured in capillary blood using YSI 1500 SPORT (YSI Inc., Yellow Spring Instruments Ohio, USA) as described [27]. Blood lactate was measured before and after the four jumping series, except for the jumping series immediately after the strength training. Lactate was measured only after the jumping series immediately after the strength training to avoid delay between the strength training and jumping series.

\section{Statistics}

Data are presented as mean \pm SEM. Maximal jumping heights between CAF and PLA were compared by $T$ test. 
Jumping heights in jump series and lactate concentrations with and without caffeine intake were compared with repeated measurements ANOVA with LSD as post hoc test. $P<0.05$ are considered as significant differences. Effect size was expressed as Cohen's $d$ [29]. Formula for calculation of Cohen's $d=\left(M_{2}-M_{1}\right) / \mathrm{SD}_{\text {pooled }}$, where $\mathrm{SD}_{\text {pooled }}=\sqrt{ }\left(\left(\mathrm{SD}_{1}^{2}+\mathrm{SD}_{2}^{2}\right) / 2\right)$. Abbreviations: $M_{1}$ and $M_{2}$ are means of the two tests, and $\mathrm{SD}_{1}$ and $\mathrm{SD}_{2}$ are standard deviations for the two tests compared.

\section{Results}

Caffeine intake increased maximal CMJ from $34.4 \pm 1.0$ to $36.7 \pm 1.3 \mathrm{~cm}$ (Fig. 1; ANOVA $P<0.01$ ). The effect size (Cohen's $d$ ) was 0.68 . Caffeine also increased CMJ at the 5-jump series with start every $7 \mathrm{~s}$ (Fig. 2b; ANOVA $P<0.05)$. The strength training session decreased CMJ to $28.8 \pm 1.0$ and $30.7 \pm 1.2 \mathrm{~cm}$ in PLA and CAF (no significant difference; Fig. 2c). Jump height during the 5 -jump series also decreased immediately after strength training, and caffeine did not increase jumping height (Fig. 1d). During the first 5 min of recovery, jumping height increased to $31.0 \pm 1.0$ and $31.7 \pm 0.9 \mathrm{~cm}$ in PLA and CAF, respectively (Fig. 2e, f), but there were no effect of caffeine on jumping height. From 5 to 15 min of recovery no further increase in jumping heights was observed (Fig. 2). Lactate was $\sim 1 \mathrm{mmol} / \mathrm{L}$ at rest and increased to $\sim 2.5 \mathrm{mmol} / \mathrm{L}$ during the warm up before the first jump (Fig. 2). Lactate during the strength training session increased to $6.97 \pm 1.20$ and $7.77 \pm 0.54 \mathrm{mmol} / \mathrm{L}$ in PLA and CAF, respectively ( $T$-test: $P=0.19$ ). Blood lactate decreased gradually during the 15 min recovery period, where the jumps were performed (Fig. 3). Importantly, blood lactate was higher in the recovery period after caffeine intake compared to placebo (repeated measurements ANOVA: $P<0.05)$.

Analysis of force production during vertical jumping showed no significant differences at any time point (Fig. 4b). The maximal force production was reduced immediately after the strength training session but recovered after $5 \mathrm{~min}$. The duration of force production increased immediately after the strength training session (Fig. 4c), supporting lower force production at higher speed (increased curvature of the force-velocity relationship). After 15 min recovery, vertical jumping height remained reduced, whereas both maximal force production and duration of force production had recovered (Fig. 4b-d).

\section{Discussion}

The main finding of the present study was that caffeine increased maximal vertical jumping height by $\sim 2 \mathrm{~cm}$. However, caffeine did not increase jumping height after three sets of heavy leg press strength training. Of note, blood lactate was higher in the recovery period after intake of caffeine indicating more severe effort during the strength training. The $\sim 2 \mathrm{~cm}$ increase in maximal jumping height will have large impact in sport competitions.

Caffeine improves performance in a number of different experimental situations. We have shown that caffeine increases double-poling performance in exercise tests lasting from 4 min until $30 \mathrm{~min}$ [30-32]. Caffeine lowers perceived exertion at submaximal loads during endurance exercise [11, 16, 30-32] and increases anaerobic capacity [6]. In the present study, the effect of caffeine on jumping height was consistent and caffeine increased CMJ on the two first and mean CMJ height during the three maximal jumps. Moreover, caffeine increased mean jumping heights at the 5 -jump series with $7 \mathrm{~s}$ between jumps prior to the strength training.

In many sports, like soccer, handball and volleyball, jumping height is a valuable physical quality, and maximal strength contributes to development of power and speed [22]. In line with this, many athletes conduct strength training to improve jumping height [17]. Importantly, the participants had experience with both strength and jump training, which allow reliable tests. In the present study, coefficient of variation $(\mathrm{CV})$ for maximal jumping height between the two first maximal jumps was $1.1 \%$, which allows detecting small effects. The effect size for maximal jumping height was moderate $(0.68)$ and the fact that caffeine increased jumping performance has practical implications in sports like high jump, where $\sim 2 \mathrm{~cm}$ increase during competitions may have dramatic effects at the final result. Recent studies support the finding that caffeine increases maximal jumping height $[3,24,41]$. Importantly, Perez-Lopez et al. found that caffeine increased performance at a number of test evaluating explosive strength including counter movement jumping height [24]. Increased jumping height has also been found in volleyball players [41] and basketball players [25] after intake of caffeine. The present study supports that caffeine improves jumping height, which requires fast and high force development.

Caffeine has several mechanisms of action, which may mediate the improved performance. Caffeine binds to and inhibits adenosine receptors at low physiological concentrations [9]. Adenosine receptors are expressed on most cell types, and caffeine has effects on both the nervous system and peripheral tissue. Caffeine has many effects on the nervous system, and caffeine increases spinal excitability [38], 

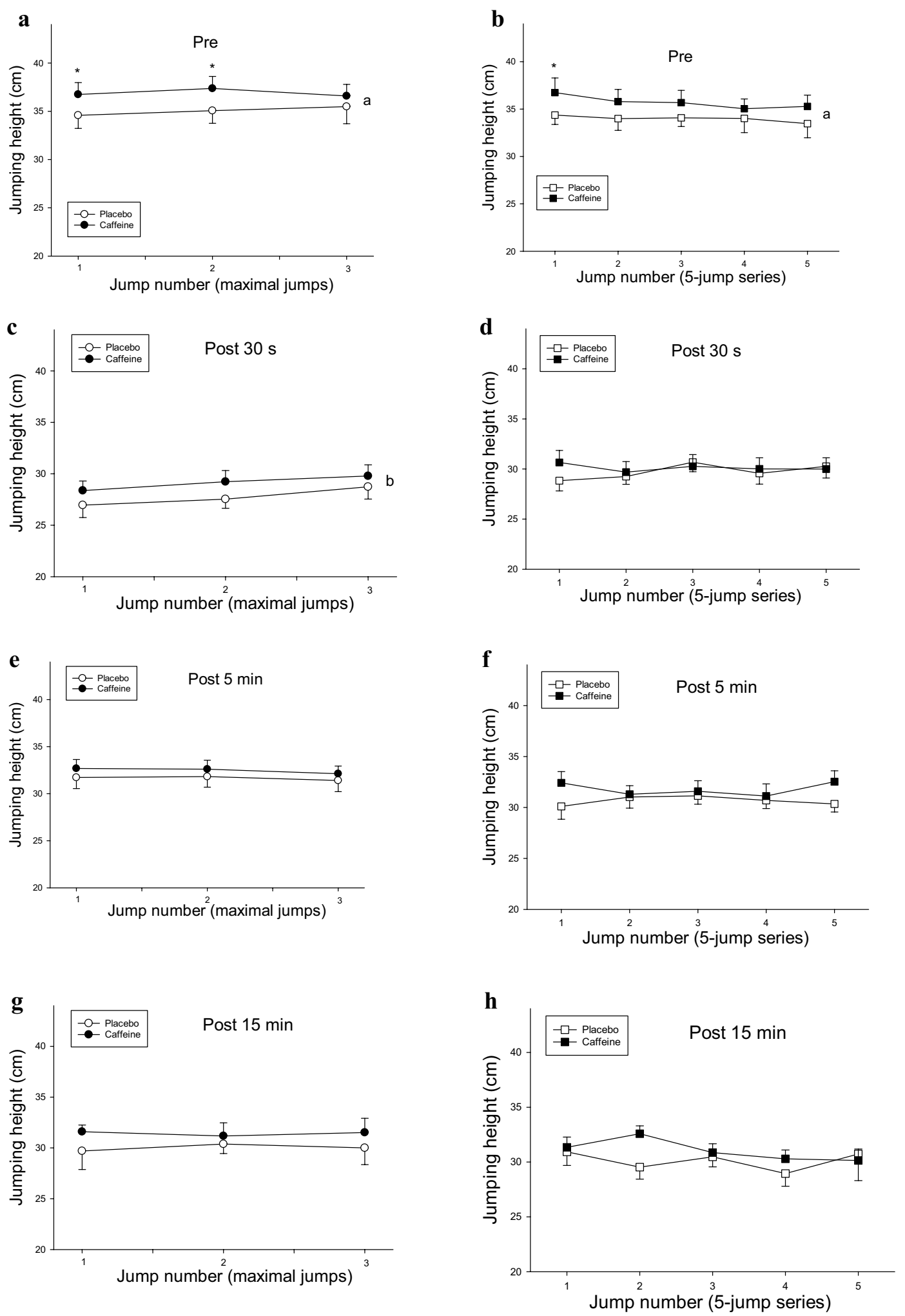

Fig. 2 Jumping height with and without caffeine intake before strength training. a Jumping height at the three maximal jumps with 30 s rest between jumps. $N=6$ except for jump 3 , where $n=5$ for placebo. b Jumping height at 5-jump series with $7 \mathrm{~s}$ rest between

jumps. $N=6$ except for jump $4(n=5)$ and jump $5(n=4)$ in placebo. Repeated measurement ANOVA treatment effect; a $P<0.05$; b repeated measurement ANOVA time effect. Post hoc (LSD) $* P<0.05$ comparing placebo and caffeine 


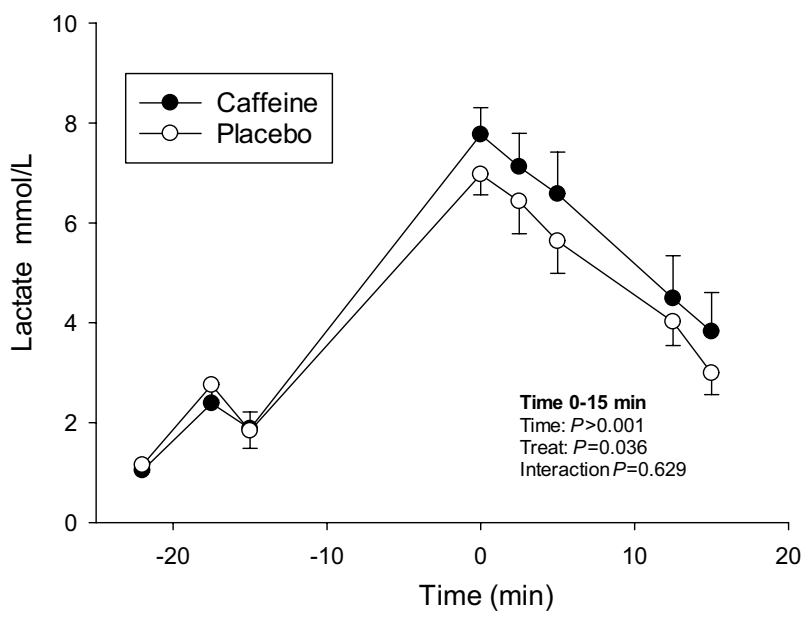

Fig. 3 Effect of caffeine on blood lactate before and after a $15 \mathrm{~min}$ session of heavy leg press strength training. The strength training was performed from 0 to $15 \mathrm{~min}$ and jumps series were performed before and during the recovery period. $N=6$ for all data points except Placebo 0 and $17 \mathrm{~min}$ and Caffeine $22 \mathrm{~min}$, where $n=5$
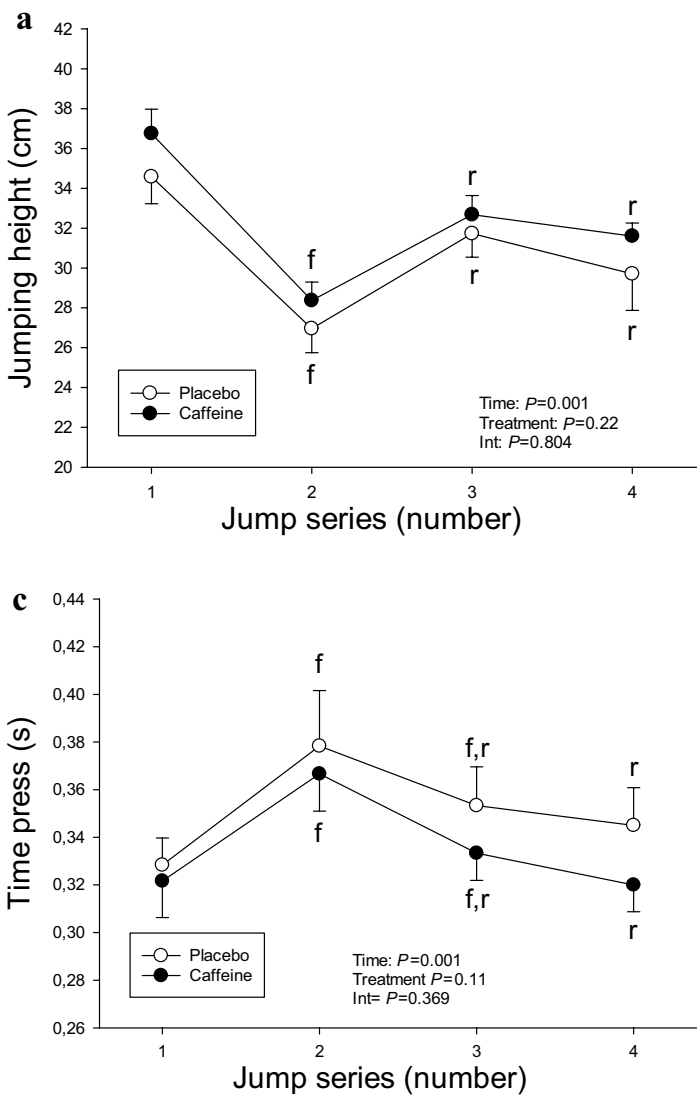

Fig. 4 Jumping heights, maximal forces and jump time in the first jump in the four jumping series before and after a session of strength training session. Jump series were conducted before (1), and $30 \mathrm{~s} \mathrm{(2),}$ 5 min (3) and 15 min (4) after a session of strength training. a Maximal jumping height in the first jump in the jumping series. b Maximal but caffeine affects several systems in the brain, which can explain increased jumping heights [23]. In the present study, jumping heights were calculated with a force platform and the speed leaving the ground determines the heights. We were unable to detect any significant effects of caffeine on maximal force production, although the statistical analyses indicated a higher maximal force production (ANOVA; $P=0.059$ ) and faster press time in jumps (ANOVA; $P=0.11)$. Higher force production in the jump phase can result from recruitment of more motor units or higher firing rate [15]. In the present study, EMG was not recorded, but there are several mechanisms that could explain the elevated jumping height.

Caffeine has direct effect on skeletal muscles [36], and it is well-documented that caffeine increases $\mathrm{Ca}^{2+}$ release via binding to ryanodine receptor [33]. In humans, physiological concentrations of caffeine increases force production during nerve stimulation, indicating that caffeine has a direct effect on skeletal [35]. Caffeine has been reported to preserve force production longer during isometric contraction without increasing motor neuron firing rate [21], which

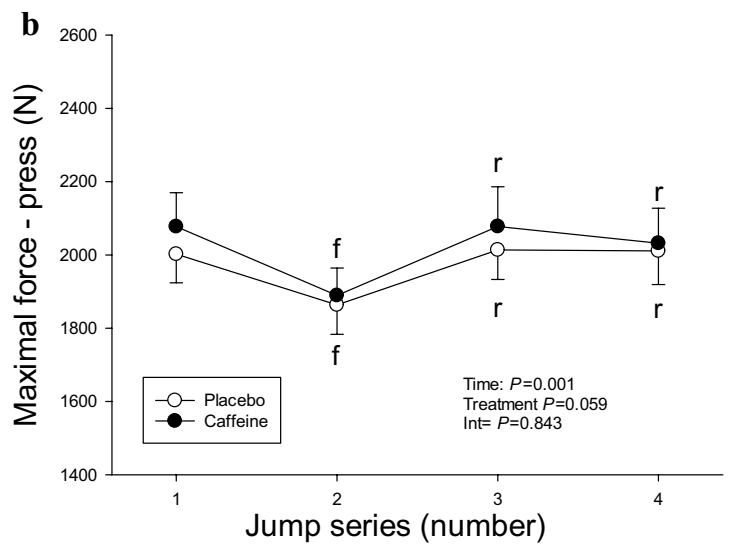

force in the press phase of first jump in the jumping series. c Time used in the press phase of the jump in the first jump in the jumping series. Repeated measurement ANOVA time effect. $f: P<0.05$ compared to Pre (1). $r: P<0.05$ compared to $30 \mathrm{~s}$ after the strength training 
indicates direct effects on skeletal muscles. In particular, the fact that caffeine stimulates $\mathrm{Ca}^{2+}$ release [33] could contribute to elevated force and jumping height.

In the present study, the effect of caffeine was observed before the heavy strength training session in leg press, but no significant effect was observed immediately after and during the recovery period. The strength training session decreased maximal CMJ by $\sim 8 \mathrm{~cm}$ immediately after the strength training in both PLA and CAF, but jumping height was not significantly higher after caffeine intake. These data show that jumping height decreases substantial after maximal effort strength training, and the jumping height remained significantly reduced during the 15 min recovery despite jumping height increased significantly during the first $5 \mathrm{~min}$ recovery. Caffeine intake increases performance, which results in more severe fatigue and elevated physiological markers of stress [32]. It has been reported that twitch-force was lower after a 4-km time trial with intake of caffeine compared to placebo [7]. In the present study, blood lactate was higher in the recovery period after caffeine ingestion compared to placebo, indicating that more work was done during the strength training session.

The mechanisms for the large decrease in jumping height after the strength training are not clear. Blood lactate is normally higher after caffeine ingestion when endurance exercise performance tests are conducted [30-32]. In the present study, the strength training session was though and increased lactate concentration to $\sim 8 \mathrm{mmol} / \mathrm{L}$, and blood lactate was higher in the recovery period after intake of caffeine, supporting that larger effort during the strength training session after caffeine ingestion, which might be the reason why caffeine no longer improved jumping height. However, blood lactate decreased during the recovery period and lactate was only slightly elevated after 15 min recovery, whereas jumping height did not increase between 5 and 15 min recovery.

To investigate the mechanism for the reduction in maximal jumping height after the short maximal effort strength training session, maximal force production and the duration of force production during jumping were investigated. Fatigue reduces maximal force, maximal contraction velocity and curvature of the force-velocity relationship, which will all contribute to reduced power production $[18,20]$. In the present study, maximal force production decreased immediately after the strength training session, which have contributed to the reduction in vertical jumping height. Immediately after the strength training, the duration of the force production was also longer, indicating that lower force was produces at higher velocities during the jump. Maximal force production had recovered after five recovery, which is supported by other studies [10]. However, the duration of jump remained longer, suggesting that the maximal effort strength training primarily affects force development at higher speed. This idea agrees with the fact that fatigue increases the curvature of the force-velocity curve [20]. After 15 min recovery, no difference in maximal force or duration of force production was observed, agreeing with increased curvature of the force-velocity curve, and lower force production at higher contraction speeds [18], resulting in lower jumping height. Caffeine did not influence the vertical jumping height during the recovery period, although duration of force production time tended to be lower after caffeine intake.

Interestingly, Glaister et al. reported that caffeine improved power during the first three sprints, whereas no effect was observed during the following seven sprints [12]. This suggests that one has to "pay the price" for the better performance during the first exercise tasks after caffeine intake later on. It has also been reported that caffeine increases 1RM in back squat [14], and the higher performance requires higher energy production and metabolic stress. The higher effort during physical activity after intake of caffeine, compared to placebo, will cause exhaustion and muscles damage, supported by what we previously reported that higher plasma creatine kinase (CK) was observed the day after caffeine intake prior to the performance test [32]. Therefore, it is not surprising that caffeine did not increase performance after a hard strength training session.

There are some limitations and strengths to keep in mind when the data in the present study are interpreted. It is a limitation that the number of participants are low, since low number of participants is associated with risk for type II errors. In the present study, the investigations of force production in the jumps did not show significant effect of caffeine. This lack of significant effect could be a type II error and these data need to be interpreted with caution and this question need to investigated more carefully. However, the effect of caffeine prior to the strength training session was consistent. Moreover, it is a strength that the participants had experience from both jump and strength training.

In conclusion, caffeine improved maximal vertical jump height and jump height during a 5-jump series. Jumping height decreased by $\sim 8 \mathrm{~cm}$ immediately after three series of heavy leg press strength training and no significant effect of caffeine was observed. After 5 and 15 min recovery of recovery, jumping height was still reduced and no significant effect of caffeine on recovery was observed. The data suggest that caffeine can improve performance in competitions like high jumping.

Acknowledgements Open Access funding provided by Norwegian School Of Sport Sciences - The Library. We thank the participants for their dedicated effort and Hans K Stadheim for mixing the caffeine drinks. 


\section{Compliance with Ethical Standards}

Conflict of interest The authors declare no conflict of interests.

Open Access This article is licensed under a Creative Commons Attribution 4.0 International License, which permits use, sharing, adaptation, distribution and reproduction in any medium or format, as long as you give appropriate credit to the original author(s) and the source, provide a link to the Creative Commons licence, and indicate if changes were made. The images or other third party material in this article are included in the article's Creative Commons licence, unless indicated otherwise in a credit line to the material. If material is not included in the article's Creative Commons licence and your intended use is not permitted by statutory regulation or exceeds the permitted use, you will need to obtain permission directly from the copyright holder. To view a copy of this licence, visit http://creativecommons.org/licenses/by/4.0/.

\section{References}

1. Bazzucchi I, Felici F, Montini M, Figura F, Sacchetti M. Caffeine improves neuromuscular function during maximal dynamic exercise. Muscle Nerve. 2011;43(6):839-44.

2. Behrens M, Mau-Moeller A, Weippert M, Fuhrmann J, Wegner K, Skripitz R, Bader R, Bruhn S. Caffeine-induced increase in voluntary activation and strength of the quadriceps muscle during isometric, concentric and eccentric contractions. Sci Rep. 2015;5:10209.

3. Bloms LP, Fitzgerald JS, Short MW, Whitehead JR. The effects of caffeine on vertical jump height and execution in collegiate athletes. J Strength Cond Res. 2016;30(7):1855-61.

4. Buckley JP, Borg GA. Borg's scales in strength training; from theory to practice in young and older adults. Appl Physiol Nutr Metab. 2011;36(5):682-92.

5. Cappelletti S, Piacentino D, Sani G, Aromatario M. Caffeine: cognitive and physical performance enhancer or psychoactive drug? Curr Neuropharmacol. 2015;13(1):71-88.

6. Davis JK, Green JM. Caffeine and anaerobic performance: ergogenic value and mechanisms of action. Sports Med. 2009;39(10):813-32.

7. Felippe LC, Ferreira GA, Learsi SK, Boari D, Bertuzzi R, LimaSilva AE. Caffeine increases both total work performed above critical power and peripheral fatigue during a 4-km cycling time trial. J Appl Physiol. 2018;1985(124):1491-501.

8. Foukas LC, Daniele N, Ktori C, Anderson KE, Jensen J, Shepherd PR. Direct effects of caffeine and theophylline on p110 delta and other phosphoinositide 3-kinases. Differential effects on lipid kinase and protein kinase activities. J Biol Chem. 2002;277(40):37124-30.

9. Fredholm BB, Battig K, Holmen J, Nehlig A, Zvartau EE. Actions of caffeine in the brain with special reference to factors that contribute to its widespread use. Pharmacol Rev. 1999;51(1):83-133.

10. Froyd C, Beltrami FG, Noakes TD. Neuromuscular fatigue at task failure and during immediate recovery after isometric knee extension trials. Sports (Basel). 2018;6(4):156.

11. Glaister M, Gissane C. Caffeine and physiological responses to submaximal exercise: a meta-analysis. Int J Sports Physiol Perform. 2018;13(4):402-11.

12. Glaister M, Howatson G, Abraham CS, Lockey RA, Goodwin JE, Foley P, McInnes G. Caffeine supplementation and multiple sprint running performance. Med Sci Sports Exerc. 2008;40(10):1835-40.
13. Grgic J. Caffeine ingestion enhances Wingate performance: a meta-analysis. Eur J Sport Sci. 2017;18(2):1-7.

14. Grgic J, Mikulic P. Caffeine ingestion acutely enhances muscular strength and power but not muscular endurance in resistancetrained men. Eur J Sport Sci. 2017;17(8):1029-36.

15. Hounsgaard J. Motor neurons. Compr Physiol. 2017;7:463-84.

16. Ivy JL, Costill DL, Fink WJ, Lower RW. Influence of caffeine and carbohydrate feedings on endurance performance. Med Sci Sports. 1979;11:6-11.

17. Jensen J, Jacobsen ST, Hetland S, Tveit P. Effect of combined endurance, strength and sprint training on maximal oxygen uptake, isometric strength and sprint performance in female elite handball players during a season. Int J Sports Med. 1997;18(5):354-8.

18. Jones DA. Changes in the force-velocity relationship of fatigued muscle: implications for power production and possible causes. J Physiol. 2010;588(Pt 16):2977-86.

19. Kolnes AJ, Ingvaldsen A, Bolling A, Stuenaes JT, Kreft M, Zorec $\mathrm{R}$, Shepherd PR, Jensen J. Caffeine and theophylline block insulin-stimulated glucose uptake and PKB phosphorylation in rat skeletal muscles. Acta Physiol (Oxf). 2010;200(1):65-74.

20. Kristensen AM, Nielsen OB, Pedersen TH, Overgaard K. Fatiguing stimulation increases curvature of the force-velocity relationship in isolated fast-twitch and slow-twitch rat muscles. J Exp Biol. 2019;222(Pt 15):jeb204545.

21. Meyers BM, Cafarelli E. Caffeine increases time to fatigue by maintaining force and not by altering firing rates during submaximal isometric contractions. J Appl Physiol. 2005;1985(99):1056-63.

22. Moss BM, Refsnes PE, Abildgaard A, Nicolaysen K, Jensen J. Effects of maximal effort strength training with different loads on dynamic strength, cross-sectional area, load-power and load-velocity relationships. Eur J Appl Physiol Occup Physiol. 1997;75(3):193-9.

23. Nehlig A, Daval JL, Debry G. Caffeine and the central nervous system: mechanisms of action, biochemical, metabolic and psychostimulant effects. Brain Res Brain Res Rev. 1992;17(2):139-70.

24. Perez-Lopez A, Salinero JJ, Abian-Vicen J, Valades D, Lara B, Hernandez C, Areces F, Gonzalez C, Del CJ. Caffeinated energy drinks improve volleyball performance in elite female players. Med Sci Sports Exerc. 2015;47(4):850-6.

25. Puente C, Abian-Vicen J, Salinero JJ, Lara B, Areces F, Del CJ. Caffeine improves basketball performance in experienced basketball players. Nutrients. 2017;9(9):1033.

26. Rush JW, Spriet LL. Skeletal muscle glycogen phosphorylase a kinetics: effects of adenine nucleotides and caffeine. J Appl Physiol. 2001;1985(91):2071-8.

27. Rustad PI, Sailer M, Cumming KT, Jeppesen PB, Kolnes KJ, Sollie O, Franch J, Ivy JL, Daniel H, Jensen J. Intake of protein plus carbohydrate during the first two hours after exhaustive cycling improves performance the following day. PLoS One. 2016;11(4):e0153229.

28. Sabol F, Grgic J, Mikulic P. The effects of three different doses of caffeine on jumping and throwing performance: a randomized, double-blind, crossover study. Int J Sports Physiol Perform. 2019;14:1-25.

29. Sawilowsky SS. New effect size rules of thumb. J Mod Appl Stat Methods. 2009;8:597-9.

30. Stadheim HK, Kvamme B, Olsen R, Drevon CA, Ivy JL, Jensen J. Caffeine increases performance in cross-country double-poling time trial exercise. Med Sci Sports Exerc. 2013;45(11):2175-83.

31. Stadheim HK, Nossum EM, Olsen R, Spencer M, Jensen J. Caffeine improves performance in double poling during acute exposure to 2000-m altitude. J Appl Physiol. 2015;1985(119):1501-9. 
32. Stadheim HK, Spencer M, Olsen R, Jensen J. Caffeine and performance over consecutive days of simulated competition. Med Sci Sports Exerc. 2014;46(9):1787-96.

33. Tallis J, Duncan MJ, James RS. What can isolated skeletal muscle experiments tell us about the effects of caffeine on exercise performance? Br J Pharmacol. 2015;172(15):3703-13.

34. Tallis J, Yavuz HCM. The effects of low and moderate doses of caffeine supplementation on upper and lower body maximal voluntary concentric and eccentric muscle force. Appl Physiol Nutr Metab. 2018;43(3):274-81.

35. Tarnopolsky M, Cupido C. Caffeine potentiates low frequency skeletal muscle force in habitual and nonhabitual caffeine consumers. J Appl Physiol. 2000;1985(89):1719-24.

36. Tarnopolsky MA. Effect of caffeine on the neuromuscular system-potential as an ergogenic aid. Appl Physiol Nutr Metab. 2008;33(6):1284-9.
37. Vikmoen O, Raastad T, Seynnes O, Bergstrom K, Ellefsen S, Ronnestad BR. Effects of heavy strength training on running performance and determinants of running performance in female endurance athletes. PLoS One. 2016;11(3):e0150799.

38. Walton C, Kalmar J, Cafarelli E. Caffeine increases spinal excitability in humans. Muscle Nerve. 2003;28(3):359-64.

39. Warren GL, Park ND, Maresca RD, McKibans KI, MillardStafford ML. Effect of caffeine ingestion on muscular strength and endurance: a meta-analysis. Med Sci Sports Exerc. 2010;42(7):1375-87.

40. Xu F, Stevens RC. Trapping small caffeine in a large GPCR pocket. Structure. 2011;19(9):1204-7.

41. Zbinden-Foncea H, Rada I, Gomez J, Kokaly M, Stellingwerff T, Deldicque L, Penailillo L. Effects of caffeine on countermovement-jump performance variables in elite male volleyball players. Int J Sports Physiol Perform. 2018;13(2):145-50. 